\title{
A Soluble Epoxide Hydrolase Inhibitor Upregulated KCNJ12 and KCNIP2 by Downregulating MicroRNA-29 in a Mouse Model of Myocardial Infarction
}

\author{
Xiao-Jun Zhang, ${ }^{1 *}$ Cai-Xiu Liao,, $2^{1 *}$ Kai-Jun Sun, ${ }^{1}$ Lei-Ling Liu, ${ }^{1}$ Dan-Yan $\mathrm{Xu}{ }^{1}$ \\ ${ }^{1}$ Department of Internal Cardiovascular Medicine, The Second Xiangya Hospital, Central South University, Changsha; ${ }^{2}$ Department \\ of Geratology, The Third Hospital of Changsha, Changsha, Hunan, China
}

\section{ABSTRACT}

Background: Soluble epoxide hydrolase inhibitors (sEHi) have anti-arrhythmic effects, and we previously found that the novel sEHi t-AUCB (trans-4[-4-(3-adamantan-1-yl-ureido)cyclohexyloxy]-benzoic acid) significantly inhibited ventricular arrhythmias after myocardial infarction (MI). However, the mechanism is unknown. It's known that microRNA-29 (miR-29) participates in the occurrence of arrhythmias. In this study, we investigated whether sEHi t-AUCB was protective against ischemic arrhythmias by modulating miR-29 and its target genes KCNJ12 and KCNIP2.

Methods: Male 8-week-old C57BL/6 mice were divided into five groups and fed distilled water only or distilled water with t-AUCB of different dosages for seven days. Then, the mice underwent MI or sham surgery. The ischemic region of the myocardium was obtained 24 hours after MI to detect miR-29, KCNJ12, and KCNIP2 mRNA expression levels via real-time PCR and KCNJ12 and KCNIP2 protein expression levels via western blotting.

Results: MiR-29 expression levels were significantly increased in the ischemic region of MI mouse hearts and the mRNA and protein expression levels of its target genes KCNJ12 and KCNIP2 were significantly decreased. T-AUCB prevented these changes dose-dependently.

Conclusion: The sEHi t-AUCB regulates the expression levels of miR-29 and its target genes KCNJ12 and KCNIP2, suggesting a possible mechanism for its potential therapeutic application in ischemic arrhythmia.

\section{INTRODUCTION}

Ischemic arrhythmias, especially the ventricular arrhythmias, are the main reasons for sudden death in acute

Received April 8, 2020; received in revised form May 14, 2020; accepted May $15,2020$.

*Both authors contributed equally to this work.

This work was supported by the National Nature Scientific Funding of China (No.81672264, No.81871858).

Correspondence: Dan-Yan Xu, 139 Middle Renmin Road, Department of Internal Cardiovascular Medicine, The Second Xiangya Hospital, Central South University, Changsha, Hunan, China 410011; +86-731-85292100; fax: +86-731-85295407 (e-mail address: xudanyan02@csu.edu.cn). myocardial infarction (MI). As conventional treatment relies on the classic anti-arrhythmic drugs with proarrhythmic potential and limited effectiveness, it is crucial to discover more effective anti-arrhythmic drugs.

Epoxyeicosatrienoic acids (EETs) are the main metabolites of arachidonic acids catalyzed by cytochrome P450 (CYP450) epoxidases [Spector 2009]. A potent cardioprotective role for EETs has been established in the past decade, which was explained by antihypertensive and antihypertrophic effects in the cardiovascular system [He 2016; Červenka 2018; Yeboah 2016]. However, EETs are unstable and quickly degrade into inactive dihydroxyeicosatrienoic acids (DHETs) in the presence of soluble epoxide hydrolase (sEH) [Inceoglu 2007]. Soluble epoxide hydrolase inhibitors (sEHi) could enhance the beneficial effects of EETs by elevating endogenous EET levels [Xu 2013; Guo 2018]. Importantly, increasing studies have found that sEHi could prevent and reduce the occurrence of arrhythmias both in murine models with hypertrophy and $\mathrm{MI}$ and serve as promising drug for anti-arrhythmia [Červenka 2018; [Gui 2018; Liu 2017; Gui 2017; Sirish 2016]. However, the underlying mechanism is unknown.

Previously, we found that the sEHi suppressed the susceptibility to increased ventricular arrhythmias after MI through the modulation of microRNA-1 (miR-1) and its target genes, which encode various ion channel proteins associated with arrhythmias [Liu 2017]. In preliminary study, higher microRNA-29 (miR-29) expression levels were tested in the ischemic area post-MI through miRNA microarray technology. Meanwhile, via miRBase and miRANDA target prediction program, it has been predicted that the target genes of miR-29 included KCNJ12 (potassium channel inwardly rectifying subfamily J member 12) and KCNIP2 (voltage-gated potassium channel-interacting protein 2), which both encode important potassium channel-related proteins involved in arrhythmia. As miR-1 and miR-29 have the same proarrhythmic effects in the heart [Dawson 2013; Boštjančič 2014; Zhao 2016], the present research aimed to investigate whether sEHi trans-4[-4-(3-adamantan-1-yl-ureido)-cyclohexyloxy]-benzoic acid (t-AUCB) could inhibit ischemic arrhythmias by modulating miR-29 and its target genes KCNJ12 and KCNIP2.

\section{METHODS}

\section{Mice and MI Model}

All animal protocols were approved by the Animal Research Committee, Central South University, Hunan, 
China. Male 8-week-old C57BL/6 mice $(26.07 \pm 0.55 \mathrm{~g})$ were obtained from the Medical Experimental Animal Center of Hunan Province. The mice were randomly divided into five groups $(\mathrm{n}=6)$ : (i) Sham, (ii) MI, (iii) 0.001 $\mathrm{mg} / \mathrm{L} \mathrm{t}-\mathrm{AUCB}+\mathrm{MI}$, (iv) $0.01 \mathrm{mg} / \mathrm{L} \mathrm{t}-\mathrm{AUCB}+\mathrm{MI}$, (v) 0.1 $\mathrm{mg} / \mathrm{L} \mathrm{t}-\mathrm{AUCB}+\mathrm{MI}$. t-AUCB $(0.01,0.1$, and $1 \mathrm{mg} / \mathrm{L})$ was administered to mice orally in drinking water for 7 days prior to surgery. The mice were anesthetized with $1.5 \%$ isoflurane inhalation; the trachea was intubated and the mice were ventilated with a small respirator (MiniVent Type 845, Hugo Sachs Elektronik, March, Germany). The left anterior descending coronary artery was ligated via left thoracotomy between the third and fourth ribs, as described previously [Ulu 2008]. Sham-operated animals underwent the same procedure without occlusion of the coronary artery.

\section{Materials}

The t-AUCB was a gift from Prof. Bruce D. Hammock (University of California, Davis, CA, USA). Rabbit anti-mouse KCNIP2 and $\beta$-actin antibodies were purchased from Abcam (Cambridge, UK) and rabbit anti-mouse KCNJ12 (or Kir2.2) was purchased from GeneTex (Irvine, CA, USA). TaqMan MicroRNA RT Kit, TaqMan MicroRNA-29b assays, TaqMan U6 snRNA assay, and TaqMan Universal PCR Master Mix were purchased from Applied Biosystems (NY, USA).

\section{Tissue Samples}

Mice were killed by cervical dislocation under anaesthesia with $1.5 \%$ isoflurane $24 \mathrm{~h}$ after MI and the hearts were obtained. Ventricular tissues from the border zone of the infarcted region were dissected and sliced into $2-\mathrm{mm}$ thick sections. Samples were stored at $-80^{\circ} \mathrm{C}$.

\section{Histological Analysis}

The hearts were fixed in neutralized formalin buffer for 24-48 h, and then cut transversely from the apex of the myocardium to the base. The fixed samples were dehydrated in an ethanol gradient, paraffin-embedded, and sectioned into 4- $\mu \mathrm{m}$ slices. After deparaffinization, the samples were stained with hematoxylin-eosin (HE) for histological examination.

\section{Real-Time PCR Detection of miR-29, KCNF12, and KCNIP2 mRNA}

Total RNA was isolated with TRIzol reagent (Invitrogen) from mouse hearts obtained post-MI. MiR-29 mRNA levels were analyzed by real-time reverse transcription (RT)-PCR using the TaqMan MicroRNA RT Kit (Applied Biosystems). Briefly, $10 \mathrm{ng}$ total RNA was reverse-transcribed with specific stem-loop RT primers using the RT kit according to the manufacturer's instructions. Real-time RT-PCR was performed on complementary DNA (cDNA) using specific primers designed based on the mouse miR-29 sequence. MiR-29 expression was calculated after normalization to U6.

The miR-29b primers were 5'-ACACTCCAGCTGGGTAGCACCATTTGAAATC-3' (forward) and 5'-CTCAACTGGTGTCGTGGAGTCGGCAATTCAGTTGAGAACACTGA-3' (reverse).
The U6 primers were 5'-ATGACGTCTGCCTTGGAGAAC-3' (forward) and 5'-TCAGTGTGCTACGGAGTTCAG-3' (reverse).

For KCNJ12 and KCNIP2 mRNA quantification, total RNA was extracted using TRIzol. CDNA was prepared using a RevertAid First Strand cDNA Synthesis Kit (Fermentas). SYBR Green quantitative PCR was performed. The quantitative assay was performed using Gapdh (glyceraldehyde-3-phosphate dehydrogenase) expression as the internal control. Relative expression was calculated using the comparative threshold cycle $(\mathrm{Ct})$ method $\left(2^{-\Delta \Delta C t}\right)$. Real-time RT-PCR was performed using the following primers:

KCNF12: 5'-CCTGCGTAAGAGTCACATCG-3' (forward), 5'-GCAAGATGGTGATGGGTGAT-3' (reverse);

KCNIP2: 5'-GTCTCCTTGGAAGACAGCGT-3' (forward),

5'-GCAACTCTCTGCGTGTGAAC-3' (reverse);

Gapdh: 5'-AAGCCCATCACCATCTTCCAGGAG-3' (forward), 5'-AGCCCTTCCACAATGCCAAAG-3' (reverse).

\section{Western Blotting}

Immunoblotting was performed as previously described [Liu 2013]. The primary antibodies were anti-Kir2.2 (1:1000; GeneTex) and anti-KCNIP2 (1:1000; Abcam); anti- $\beta$-actin antibody (1:1000; Abcam) was used as the internal loading control.

\section{Statistical Analysis}

Data were analyzed using the SPSS 19.0 statistical package. Results are expressed as the mean \pm SEM. One-way analysis of variance followed by Bonferroni's post-hoc test was used for multiple comparisons. All assays were performed in triplicate. $P<.05$ was considered statistically significant.

\section{RESULTS}

\section{Identification of Mouse Model of MI}

The left ventricular apex of the heart below the ligature turned pale and thin $24 \mathrm{~h}$ after MI (Figure 1, B). Hearts in the sham group had normal shape and structure (Figure 1, A). There were no degenerated or necrotic cells in the sham group (Figure 1, C). In the MI group, there were large amounts of degenerated and necrotic cells. Myocardial cells in the infarcted zone were stained a homogeneous red, and their nuclei were not visible. The myofiber arrangement was disordered (Figure 1, D).

\section{Influence of t-AUCB on miR-29 Expression in Ischemic Area of MI Mouse Hearts}

MiR-29 expression was increased by 6.3 -fold in the MI group as compared with the sham group $(\mathrm{n}=6$, all $P<.05)$ (Figure 2). T-AUCB decreased miR-29 expression dosedependently. Compared with the MI group, miR-29 expression was decreased to $67 \%, 42 \%$, and $25 \%$ in the $0.01 \mathrm{mg} / \mathrm{L}$ t-AUCB+MI group, $0.1 \mathrm{mg} / \mathrm{L}$ t-AUCB+MI group, and 1 $\mathrm{mg} / \mathrm{L} \mathrm{t}-\mathrm{AUCB}+\mathrm{MI}$ group, respectively; all differences were statistically significant $(\mathrm{n}=6$, all $P<.05)$. 

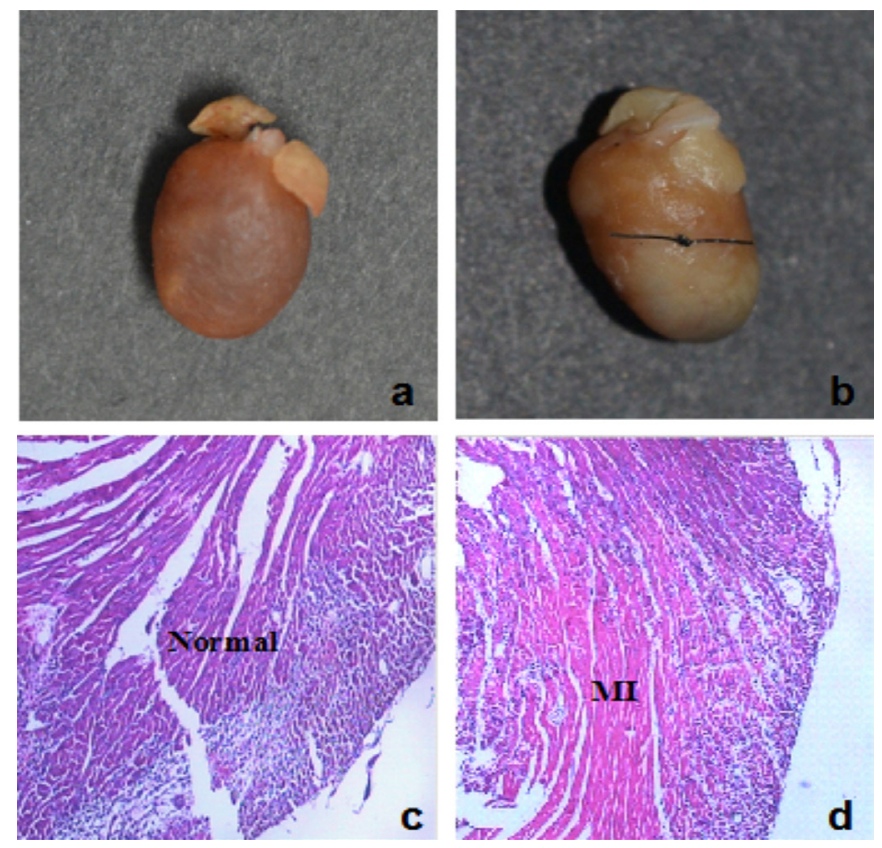

Figure 1. Identification of mouse model of MI. MI was established by ligating the left anterior descending coronary artery. The ischemic region of myocardium was cut and fixed by $4 \%$ paraformaldehyde. Gross appearance: (A) Normal heart; (B) The infarcted area of left ventricular apex below the ligature turned pale. The morphological changes were observed by HE staining. Invert micrograph $(\times 20)$ of mice myocardium; (C) Normal myocardium, marked with "Normal”; (D) Necrotic myocardium, marked with “MI."

\section{Influence of $t$-AUCB on KCNF12 and KCNIP2 mRNA Expression in the Ischemic Area Post-MI}

KCNJ12 and KCNIP2 mRNA expression was decreased to $27 \%$ and $14 \%$ in the ischemic myocardium of the MI group ( $\mathrm{n}=6$, all $P<.05)$, respectively, as compared with the sham group ( $\mathrm{n}=6$, all $P<.05$ ) (Figure 3). T-AUCB increased KCNJ12 and KCNIP2 mRNA expression dose-dependently. In the $0.01 \mathrm{mg} / \mathrm{L} \mathrm{t}-\mathrm{AUCB}+\mathrm{MI}, 0.1 \mathrm{mg} / \mathrm{L} \mathrm{t}-\mathrm{AUCB}+\mathrm{MI}$, and $1 \mathrm{mg} / \mathrm{L}$ t-AUCB+MI groups, KCNJ12 mRNA expression was 0.96 -fold, 1.8-fold, and 2.7-fold, respectively, of that in the MI group. The $0.1 \mathrm{t}-\mathrm{AUCB}+\mathrm{MI}$ and $1 \mathrm{mg} / \mathrm{L}$ t-AUCB+MI groups were statistically significantly different compared with the MI group ( $\mathrm{n}=6$, all $P<.05)$ (Figure 3, A). Compared with the MI group, KCNIP2 mRNA expression levels were increased by 3 -fold, 6.5 -fold, and 7.9-fold in the $0.01 \mathrm{mg} / \mathrm{L}$ t-AUCB+MI, $0.1 \mathrm{mg} / \mathrm{L} \mathrm{t}-\mathrm{AUCB}+\mathrm{MI}$, and $1 \mathrm{mg} / \mathrm{L}$ t-AUCB+MI groups, respectively; the differences were statistically significant $(\mathrm{n}=6$, all $P<.05)$ (Figure $3, \mathrm{~B})$.

\section{Influence of t-AUCB on KCN712 and KCNIP2 Protein in the Ischemic Area Post-MI}

Compared with the sham group, KCNJ12 and KCNIP2 protein expression was decreased to $23 \%$ and $20 \%$, respectively, in the ischemic myocardium of the MI group ( $\mathrm{n}=$ 6 , all $P<.05$ ) (Figure 4). T-AUCB increased KCNJ12 and KCNIP2 protein expression dose-dependently. In the $0.01 \mathrm{mg} / \mathrm{L} \mathrm{t}-\mathrm{AUCB}+\mathrm{MI}, 0.1 \mathrm{mg} / \mathrm{L} \mathrm{t}-\mathrm{AUCB}+\mathrm{MI}$, and 1

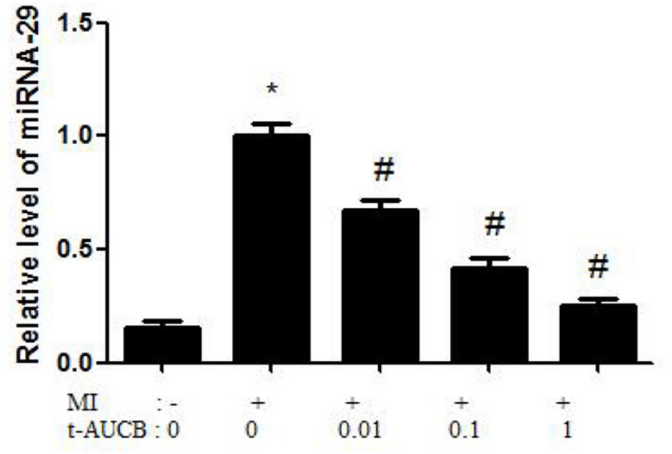

Figure 2. T-AUCB downregulated miRNA-29. Before the MI surgery or sham-operated, mice were randomized to take orally either distilled water or t-AUCB $(0.01,0.1,1 \mathrm{mg} / \mathrm{L})$ for seven days. Measurements were made 24 hours after MI. The expression of miRNA-29 was quantified by real-time PCR and the $U 6$ as reference genes to correct the difference. The quantification was performed according to the comparative method $(2-\Delta \Delta \mathrm{ct}) .{ }^{*} P \leq .05$ versus sham group, $\# P<.05$ versuss $\mathrm{MI}$ group $(\mathrm{n}=6$, all $P<.05)$.

$\mathrm{mg} / \mathrm{L}$ t-AUCB+MI groups, KCNJ12 protein expression was increased by 1.9 -fold, 2.7 -fold, and 4.6-fold, respectively, compared to the MI group (Figure 4, A). Compared with the MI group, KCNIP2 protein expression levels were increased by 2.5 -fold, 3.6-fold, and 4.6-fold in the 0.01 $\mathrm{mg} / \mathrm{L} \mathrm{t}-\mathrm{AUCB}+\mathrm{MI}, 0.1 \mathrm{mg} / \mathrm{L} \mathrm{t}-\mathrm{AUCB}+\mathrm{MI}$, and $1 \mathrm{mg} / \mathrm{L}$ t-AUCB+MI groups, respectively. All differences were statistically significant $(\mathrm{n}=6$, all $P<.05)$ (Figure $4, \mathrm{~B})$.

\section{DISCUSSION}

The present study shows that miR-29 expression levels were significantly increased in the ischemic region of myocardia from the MI group, while KCNJ12 and KCNIP2 mRNA and protein expression levels were significantly decreased. The t-AUCB dose-dependently decreased miR-29 expression levels and increased KCNJ12 and KCNIP2 mRNA and protein expression levels.

We found that miR-29 was increased in the ischemic region after MI. Our findings were consistent with that of Dong, who found that miR-29 was increased by 2.4 times in the ischemic region at $6 \mathrm{~h}$ after MI in rats [Dong 2009]. However, van Rooij et al [van Rooij 2008] found that miR-29 was decreased in the ischemic region in MI mice at 3 days and 14 days after MI. Shi et al [Shi 2010] reported that miR-29 was decreased by 2.3 times in the ischemic region at 7 days after MI in rats. Port et al also found that miR-29 was decreased in the ischemic region at 14 days after $\mathrm{MI}$ in mice [Port 2011]. The above studies appeared to be the opposite of our findings, revealing miR-29 family was dysregulated in the heart during post-MI remodeling. MiR-29 was predicted to function as inhibitors of numerous mRNAs involved in ECM 
A
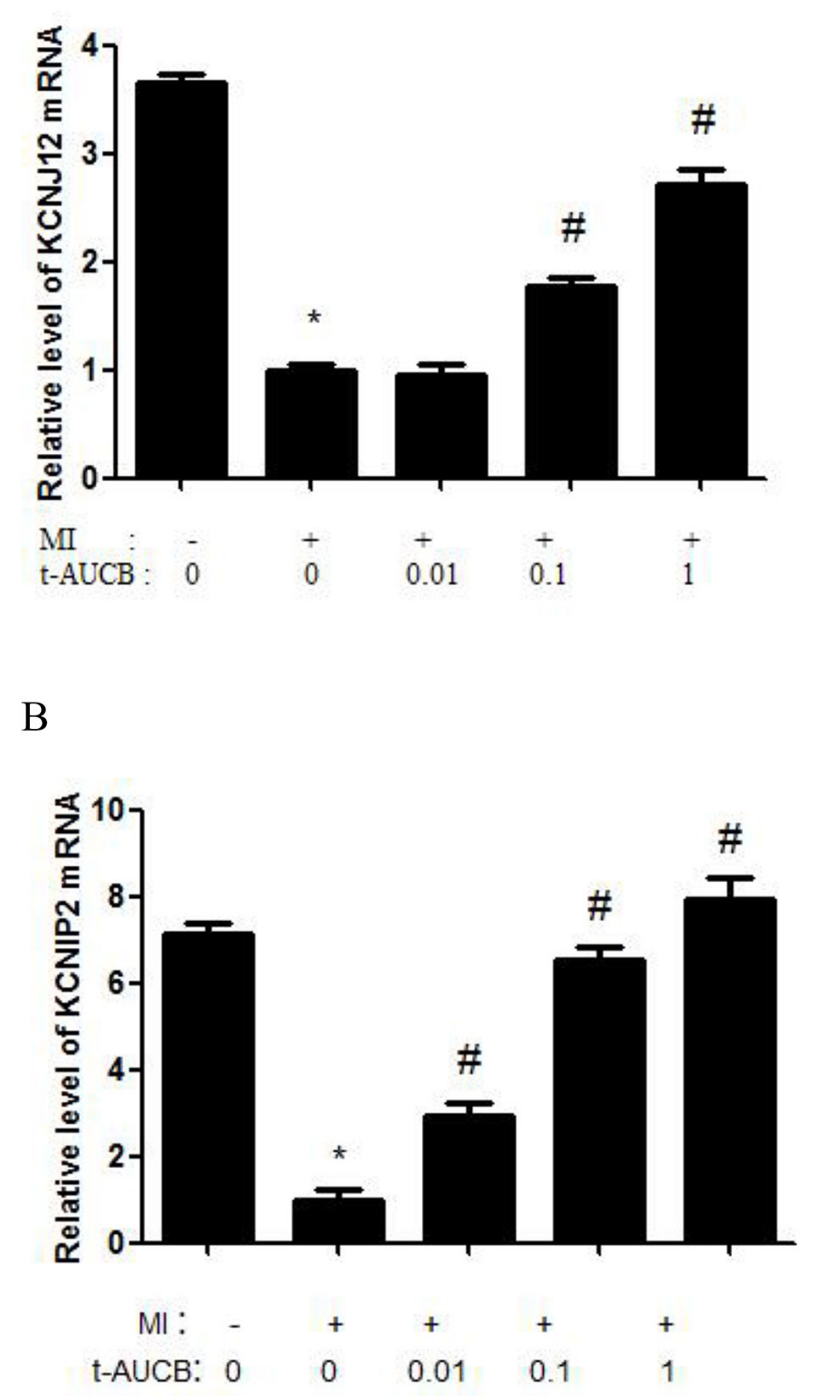

Figure 3. T-AUCB upregulated the expression of KCNJ12 and KCNIP2 mRNA. The expression of KCNJ12 and KCNIP2 mRNA were quantified by real-time PCR and the GAPDH as reference genes to correct the difference. The quantification was performed according to the comparative method $(2-\Delta \Delta \mathrm{ct}) .{ }^{*} P \leq .05$ versus sham group, $\# P<.05$ versus Ml group.

production and fibrosis [van Rooij 2008; Zhu 2013]. It is possible that the different time of detection in our study led to the differing results. We detected miR-29 expression levels in the ischemic region at $24 \mathrm{~h}$ after $\mathrm{MI}$ in mice, and Dong detected miR-29 expression levels in the ischemic region at $6 \mathrm{~h}$ after MI, the acute phase of MI. By contrast, Rooij et al, Shi et al, and Port et al detected miR-29 expression levels in the ischemic region at 3 days, 7 days, and 14 days after MI, respectively, the proliferative phase (3 days to weeks after $\mathrm{MI}$ ). In this period, the synthesis of ECM proteins such as collagen
A

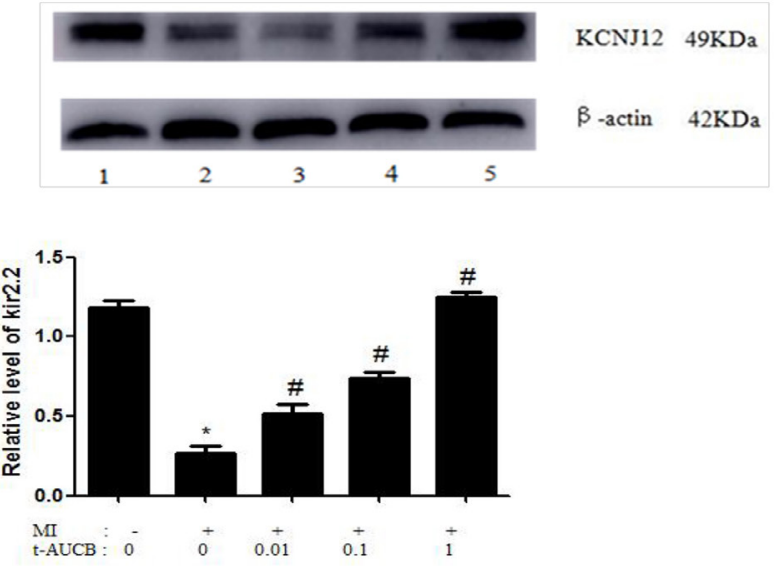

B
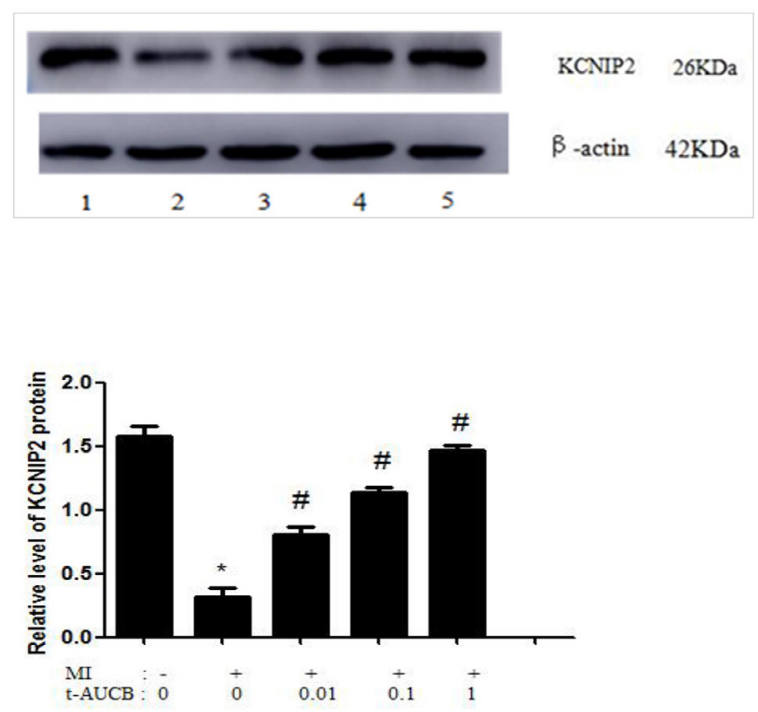

Figure 4. T-AUCB upregulated the expression of KCNJ12 and KCNIP2 protein. A, Examples of Western blot bands; B, Data are mean \pm SEM normalized to control. Quantitative assay was performed with GAPDH as an internal control. ${ }^{*} P<.05$ versus sham group; $\# P<.05$ versus $M I$ group.

and laminin was increased [Talman 2016]. Reduced miR-29 expression was correlated to the appearance of fibrotic ECM, which contributed to adverse ventricular remodeling after MI [Lin 2015]. We speculated that miR-29 expression levels in the ischemic region varied according to the detection time points after MI, so it is possible to detect miR-29 expression levels in the ischemic region at different time points after MI.

The most valuable findings from our study were that t-AUCB reversed miR-29 expression levels in the ischemic region after $\mathrm{MI}$ in mice dose-dependently. In the present study, we used $0.01 \mathrm{mg} / \mathrm{L}, 0.1 \mathrm{mg} / \mathrm{L}$, and $1 \mathrm{mg} / \mathrm{L}$ t-AUCB, which all could downregulate miR-29 expression levels in 
the ischemic region of the myocardium at $24 \mathrm{~h}$ after MI in mice, and the differences were statistically significant. Previously, our group found that the myocardium infarct size was reduced markedly in MI mice treated with t-AUCB [Gui 2017; Gui 2018]. It is clear that t-AUCB acts on miR-29 and plays a role in anti-myocardial ischemia by regulating miR-29 expression levels.

Formerly, our study group did some electrophysiological experiments that were crucial to demonstrate the anti-arrhythmic effect of t-AUCB [Liu 2017; Gui 2017]. Consistently, our previous in vitro whole-cell patch-clamp recording demonstrated that the current density of IK1 (inward rectifier $\mathrm{K}+$ current) was significantly reduced in miR-1 overexpression model of neonatal cardiac myocytes and t-AUCB could restore the IK1 current [Liu 2017]. Also, we performed surface electrocardiogram and simultaneous intracardiac electrograms from atria and ventricles from MI mice treated with or without t-AUCB. Compared with the MI group (70\%), the incidence of ventricular tachycardia was decreased to $40 \%$, and $38 \%$ in MI mice orally treated with $1 \mathrm{mg} / \mathrm{L}$ and $5 \mathrm{mg} / \mathrm{L}$ t-AUCB (all $P<.05$ ), respectively, revealing that sEHi could reduce the incidence of ventricular arrhythmias in MI mice [Gui 2017].

We found significantly decreased KCNJ12 mRNA and protein expression levels in the ischemic region after MI, and t-AUCB increased the KCNJ12 mRNA and protein expression levels dose-dependently. KCNJ12 is an ATP-sensitive inward rectifier potassium channel and encodes Kir2.2, which is involved in the formation of IK1 in the heart [Obukhov 2016]. The decreased function of Kir2.2 leads to the occurrence of electrical remodeling and the reduction of repolarization current at the end of an action potential, resulting in prolonged QT interval and action potential duration, which dramatically increase the risk of arrhythmias [Domenighetti 2007; Zaritsky 2001]. Many studies have confirmed that IK1 participated in the occurrence of ischemic arrhythmia after MI [Thireau 2015; Zhai 2017; Alexandre 2015]. Delapril, an angiotensin-converting enzyme inhibitor and mineralocorticoid receptor antagonist, inhibited the occurrence of arrhythmia simply by restoring damaged ion currents such as IK1 [Thireau 2015]. Also, we have proven that t-AUCB inhibited the occurrence of ventricular arrhythmias in mice with MI by restoring damaged IK1 [Liu 2017]. In the preliminary stage, we used a miRNA target prediction program and determined that KCNJ12 was a target gene of miR-29, and confirmed that upregulating miR-29 would downregulate KCNJ12. T-AUCB dose-dependently downregulated miR-29 and upregulated KCNJ12 in this research, which indicated that t-AUCB might regulate the expression of inward rectifier potassium channel proteins and affect the inward rectifier potassium current, and eventually play a role in anti-ischemic arrhythmias. Further experiments using miR-29 agomir or antagomir are required to verify the negative regulatory relationship between miR-29 and KCNJ12. Meanwhile, we will perform experimental studies in vitro.

We found significantly decreased KCNIP2 mRNA and protein expression levels in the ischemic region after $\mathrm{MI}$ and that t-AUCB increased the KCNIP2 mRNA and protein expression levels dose-dependently. The KCNIP2 gene encodes a member of the voltage-gated potassium (Kv) KCNIP2 family. Kv4.2 and $\mathrm{Kv} 4.3$ are the main components of transient outward potassium current (Ito), which is a potassium ion current on the myocardial cell membrane and mainly participates in the early repolarization of action potential [Deschênes 2002]; Ito abnormality is closely related to ischemic arrhythmias [Lundby 2010; Liu 2016]. Myers et al found that decreased $\mathrm{Kv} 4.2$ expression levels significantly attenuated Ito and greatly prolonged action potential duration [Myers 2015]. Accordingly, we speculated that t-AUCB might also play a role in anti-ischemic arrhythmias by recovering Ito via up-regulation of the KCNIP2 gene. In the preliminary experiment, we observed that KCNIP2 was also miR-29 target gene via miRNA target prediction program and proved initially the negative regulatory relationship between miR-29 and KCNIP2. T-AUCB dose-dependently reversed the above results, which shows that KCNIP2 might be involved in t-AUCB regulation of ischemic arrhythmias.

The present study confirms that $\mathrm{t}-\mathrm{AUCB}$ regulates the expression of the potassium ion channel-related proteins KCNJ12 and KCNIP2 to some degree. Nonetheless, we do not know how or through which signaling pathway t-AUCB regulates miR-29 in the early phase of MI. There are few articles on miR-29 regulation: some studies reported that the PI3K/Akt (phosphatidylinositol 3-kinase/ protein kinase $\mathrm{B}, \mathrm{PI} 3 \mathrm{~K} / \mathrm{Akt}$ ) signaling pathway participated in peroxisome proliferator-activated receptor-gamma (PPAR) downregulation of miR-29 expression levels in the ischemic myocardium [Ye 2010; Yasuda 2009]. Meanwhile, other studies have shown that EETs were natural ligands and endogenous agonists of PPAR $\gamma$ and activation of PPAR $\gamma$ signaling played a crucial role in EET-mediated protection in cardiomyocytes [Samokhvalov 2014]. Accordingly, we speculated that sEHi probably regulated miR-29 through the PI3K/Akt signaling pathway. Moreover, it has been indicated that other regulatory factors such as serum response factor (SRF) and myocyte enhancer factor 2 (MEF-2) were involved in EET regulation of miRNAs. These regulatory factors and cell signal transduction pathways might connect and interact with each other and function by forming a regulatory network that regulates the downstream effector proteins [Zhang 2011; Wei 2017], although the specific mechanism remains unknown, and clearly further research is needed to clarify potential links.

Additionally, many studies have found that miRNA could regulate several ion currents by regulating the expression of various ion channel proteins associated with arrhythmias, and thereby play a role in mediating arrhythmias [Gui 2018; Liu 2017; Myers 2015; Zhou 2018]. miR-29 is one of the etiological factors of ischemic arrhythmia [Dawson 2013; Boštjančič 2014], and plays an important role in the antiarrhythmia effect of sEHi. Our study indicated that miR-29 itself may serve as a novel target for the prevention and treatment of ischemic arrhythmia, which is of translational potential in future clinical studies. 


\section{Study Limitations}

We didn't include the electrocardiograph or other arrhythmia-related data, which is of paramount importance to demonstrate exactly which effects t-AUCB have on the genesis of ventricular arrhythmias in the very acute (within $24 \mathrm{hr}$ ) period; whereas, the anti-arrhythmia efficacy of t-AUCB has been proven in our previous work [Gui 2018]. Additionally, it cannot be excluded that sEHi had a protective effect and reduced infarct size and effects on miR-29 and ion channels could be secondary to this effect. Furthermore, we didn't compare the effects of t-AUCB with other sEHi to show if this is a class effect of this group of agents or possibly results from some off-target effect and is specific for t-AUCB only.

\section{Conclusion}

MiR-29 expression levels are significantly increased in the infarct border zone and the mRNA and protein expression levels of its target genes KCNJ12 and KCNIP2 are significantly decreased. At $24 \mathrm{~h}$ after MI in mice, t-AUCB dosedependently reverses these changes. However, further study is required to elucidate the mechanism of this effect.

\section{REFERENCES}

Alexandre J, Hof T, Puddu PE, et al. 2015. Rapid and MR-Independent IK1 Activation by Aldosterone during Ischemia-Reperfusion. PloS one 10:e0132592.

Boštjančič E and Glavač D. 2014. miRNome in myocardial infarction: Future directions and perspective. World J Cardiology 6:939-58.

ervenka L, Husková Z, Kopkan L, et al. 2018. Two pharmacological epoxyeicosatrienoic acid-enhancing therapies are effectively antihypertensive and reduce the severity of ischemic arrhythmias in rats with angiotensin II-dependent hypertension. Journal of hypertension 36:1326-41.

Dawson K, Wakili R, Ordög B, et al. 2013. MicroRNA29: a mechanistic contributor and potential biomarker in atrial fibrillation. Circulation 127:10.1161.

Deschênes I, DiSilvestre D, Juang GJ, et al. 2002. Regulation of Kv4.3 current by KChIP2 splice variants: a component of native cardiac I(to)? Circulation 106:423-9.

Domenighetti AA, Boixel C, Cefai D, et al. 2007. Chronic angiotensin II stimulation in the heart produces an acquired long QT syndrome associated with IK1 potassium current downregulation. Journal of molecular and cellular cardiology 42:63-70.

Dong S, Cheng Y, Yang J, et al. 2009. MicroRNA expression signature and the role of microRNA-21 in the early phase of acute myocardial infarction. J Biol Chem 284:29514-25.

Gui Y-J, Yang T, Liu Q, et al. 2017. Soluble epoxide hydrolase inhibitors, t-AUCB, regulated microRNA-1 and its target genes in myocardial infarction mice. Oncotarget 8:94635-49.

Gui Y, Li D, Chen J, et al. 2018. Soluble epoxide hydrolase inhibitors, t-AUCB, downregulated miR-133 in a mouse model of myocardial infarction. Lipids Health Dis 17:129.

Guo Y, Luo F, Zhang X, et al. 2018. TPPU enhanced exercise-induced epoxyeicosatrienoic acid concentrations to exert cardioprotection in mice after myocardial infarction. Journal of cellular and molecular medicine 22:1489-1500.
He J, Wang C, Zhu Y, et al. 2016. Soluble epoxide hydrolase: A potential target for metabolic diseases. J Diabetes 8:305-13.

Inceoglu B, Schmelzer KR, Morisseau C, et al. 2007. Soluble epoxide hydrolase inhibition reveals novel biological functions of epoxyeicosatrienoic acids (EETs). Prostaglandins \& other lipid mediators 82:42-9.

Lin Y, Sibanda VL, Zhang H-M, et al. 2015. MiRNA and TF co-regulatory network analysis for the pathology and recurrence of myocardial infarction. Scientific Rep 5:9653.

Liu L, Hayashi K, Kaneda T, et al. 2013. A novel mutation in the transmembrane nonpore region of the KCNH2 gene causes severe clinical manifestations of long QT syndrome. Heart Rhythm 10:61-7.

Liu X, Zhang Y, Du W, et al. 2016. MiR-223-3p as a novel microRNA regulator of expression of voltage-gated $\mathrm{K}+$ channel $\mathrm{Kv} 4.2$ in acute myocardial infarction. Cellular Physiol Biochem 39:102-14.

Liu Q, Zhao X, Peng R, et al. 2017. Soluble epoxide hydrolase inhibitors might prevent ischemic arrhythmias via microRNA-1 repression in primary neonatal mouse ventricular myocytes. Molecular Bio Systems 13:556-64.

Lundby A, Jespersen T, Schmitt N, et al. 2010. Effect of the I(to) activator NS5806 on cloned K(V)4 channels depends on the accessory protein KChIP2. British J Pharmacol 160:2028-44.

Myers R, Timofeyev V, Li N, et al. 2015. Feedback mechanisms for cardiac-specific microRNAs and cAMP signaling in electrical remodeling. Circ Arrhyth and Electrophysiol 8:942-50.

Obukhov AG, Scherer D, Seyler C, et al. 2016. Inhibition of cardiac kir current (IK1) by protein kinase C critically depends on PKC and Kir2.2. Plos One 11.

Port JD, Walker LA, Polk J, et al. 2011. Temporal expression of miRNAs and mRNAs in a mouse model of myocardial infarction. Physiological genomics 43:1087-95.

Samokhvalov V, Vriend J, Jamieson KL, et al. 2014. PPAR signaling is required for mediating EETs protective effects in neonatal cardiomyocytes exposed to LPS. Frontiers in pharmacology 5:242.

Shi B, Guo Y, Wang J, et al. 2010. Altered expression of microRNAs in the myocardium of rats with acute myocardial infarction. BMC cardiovascular disorders 10:11.

Sirish P, Li N, Timofeyev V, et al. 2016. Molecular mechanisms and new treatment paradigm for atrial fibrillation. Circ Arrhyth Electrophysiol 9:10.1161.

Spector AA. 2009. Arachidonic acid cytochrome P450 epoxygenase pathway. J Lipid Res 50 Suppl:S52-6.

Talman V and Ruskoaho H. 2016. Cardiac fibrosis in myocardial infarction-from repair and remodeling to regeneration. Cell and Tissue Res 365:563-81.

Thireau J, Zalvidea S, Meschin P, et al. 2015. ACE inhibitor delapril prevents $\mathrm{Ca}(2+)$-dependent blunting of IK1 and ventricular arrhythmia in ischemic heart disease. Current Mol Med 15:642-51.

Ulu A, Davis BB, Tsai H-J, et al. 2008. Soluble epoxide hydrolase inhibitors reduce the development of atherosclerosis in apolipoprotein e-knockout mouse model. J Cardiovasc Pharmacol 52:314-23.

van Rooij E, Sutherland LB, Thatcher JE, et al. 2008. Dysregulation of microRNAs after myocardial infarction reveals a role of miR-29 in cardiac fibrosis. Proc Natl Acad Sci USA 105:13027-32.

Wei X, Hou X, Li J, et al. 2017. miRNA-181a/b Regulates Phenotypes of Vessel Smooth Muscle Cells Through Serum Response Factor. DNA 
and Cell Biol 36:127-35.

Xu D-y, Davis BB, Wang Z-h, et al. 2013. A potent soluble epoxide hydrolase inhibitor, t-AUCB, acts through PPAR $\gamma$ to modulate the function of endothelial progenitor cells from patients with acute myocardial infarction. Int J Cardiol 167:1298-1304.

Yasuda S, Kobayashi H, Iwasa M, et al. 2009. .Antidiabetic drug pioglitazone protects the heart via activation of PPAR-gamma receptors, PI3kinase, Akt, and eNOS pathway in a rabbit model of myocardial infarction. Am J Physiol Heart and Circ Physiol 296:H1558-65.

Ye Y, Hu Z, Lin Y, et al. 2010. Downregulation of microRNA-29 by antisense inhibitors and a PPAR-gamma agonist protects against myocardial ischaemia-reperfusion injury. Cardiovasc Res 87:535-44.

Yeboah MM, Hye Khan MA, Chesnik MA, et al. 2016. The epoxyeicosatrienoic acid analog PVPA ameliorates cyclosporine-induced hypertension and renal injury in rats. Am J Physiol Renal Physiol 311:F576-85.

Zaritsky JJ, Redell JB, Tempel BL, et al. 2001. The consequences of disrupting cardiac inwardly rectifying $\mathrm{K}(+)$ current $(\mathrm{I}(\mathrm{K} 1))$ as revealed by the targeted deletion of the murine Kir2.1 and Kir2.2 genes. J Physiol (Lond) 2533:697-710.

Zhai X-W, Zhang L, Guo Y-F, et al. 2017. The IK1/Kir2.1 channel agonist zacopride prevents and cures acute ischemic arrhythmias in the rat. PloS one 12:e0177600.

Zhang X, Azhar G, Helms SA, et al. 2011. Regulation of cardiac microRNAs by serum response factor. Journal of biomedical science 18:15.

Zhao Y, Yuan Y and Qiu C. 2016. Underexpression of CACNA1C caused by overexpression of microRNA-29a underlies the pathogenesis of atrial fibrillation. Med Sci Monitor 22:2175-81.

Zhou S-S, Jin J-P, Wang J-Q, et al. 2018. miRNAS in cardiovascular diseases: potential biomarkers, therapeutic targets and challenges. Acta pharmacologica Sinica 39:1073-84.

Zhu J-N, Chen R, Fu Y-H, et al. 2013. Smad3 inactivation and MiR-29b upregulation mediate the effect of carvedilol on attenuating the acute myocardium infarction-induced myocardial fibrosis in rat. PloS one 8:e75557. 